\title{
The role of diet in the management of hyperlipoproteinaemias
}

\author{
By M. C. Stone, Clinical Research Unit, Leigh Infirmary, Leigh, Lancashire
}

It is no longer rational to diagnose and treat disorders of lipid transport and metabolism by measurement of serum cholesterol alone. Even the use of cholesterol and triglyceride estimations together, although permitting an adequate diagnosis in the majority of subjects (Fredrickson, Levy, Kwiterovich \& Jover, 1969), will not give as precise a resolution of hyperlipidacmias as those techniques by which the lipoproteins can be separated into three main low-density fractions. Several methods by which this separation can be achieved have been reviewed by Hatch $\&$ Lees ( 1968 ). The lipoprotein fractions are named according to the techniques by which they are separated (Table 1 ).

Table 1. Terminology used for three main low density lipoprotein fractions

Separation technique

Lipoprotein fraction Low density lipoproteins (LDL)

Very low density

lipoproteins (VLDL)

Chylomicrons

$\begin{array}{lll}\begin{array}{c}\text { Analytical } \\ \text { ultracentrifugation }\end{array} & \text { Electrophoresis } & \begin{array}{c}\text { Membrane filtration } \\ \text { and nephelometry }\end{array} \\ S_{f} \circ-20 \text { lipoproteins } & \beta \text {-Lipoproteins } & \text { Small (S) particles } \\ S_{f 20-400 \text { lipoproteins }} & \begin{array}{c}\text { Pre- } \beta \text {-lipoproteins } \\ \text { Chylomicrons }\end{array} & \begin{array}{l}\text { Medium (M) particles } \\ \text { Large (L) particles }\end{array}\end{array}$

$S_{f}$, analogous to a sedimentation contant $(S)$, but for flotation (see Masoro, I968).

Although analytical ultracentrifugation provides the most detailed quantitative analysis of lipoproteins at present available, it is not suitable for general use because of the high cost and the technical difficulty of the analyses. For practical purposes, therefore, most laboratories performing lipoprotein analysis use either electrophoresis on paper (Lees \& Hatch, I963), agarose (Noble, Hatch, Mazrimas, Lindgren, Jensen \& Adamson, 1969), or cellulose acetate (Chin \& Blankenhorn, r968).

An alternative method is the simple, inexpensive, quantitative, lipoprotein analysis described by Stone \& Thorp (1966) and modified by Stone, Thorp, Mills \& Dick (1970). Chylomicrons $\left(S_{f}>400\right)$ are separated from the smaller very low density lipoproteins $\left(S_{f} 20-400\right)$ by membrane filtration of diluted serum, and the concentration of particles in each fraction is quantified by measurement of the intensity of scattered light using the nephelometer described by Thorp, Horsfall \& Stone $(1967)$. A high correlation has been shown between the results of this method and those obtained by analytical ultracentrifugation (Stone $e t$ al. 1970). Although this technique does not provide a dircct estimation of $S_{f}$ o-20 lipoproteins, it has been shown (Stone et al. 1970) that the latter can be derived with a 
high degree of confidence $(r=0.83)$ from the nephelometric measurements and an estimation of the serum cholesterol.

\section{Lipoprotein patterns}

Several different types of lipoprotein pattern have been described by Fredrickson, Levy \& Lees (1967), based on electrophoresis together with chemical estimation of cholesterol and triglycerides, and a recent WHO memorandum (WHO, I970) has recommended this classification in a modified form. We (Stone, Thorp, Mills \& Dick, 1971) have described a quantitative classification of the lipoprotein patterns determined by membrane filtration, nephelometry, and cholesterol estimation (MNC analysis), and we have shown that the types of pattern described in this classification (the SML classification) correlate well with those described by Frederickson et al. (1967). The terminology and concentration limits for five

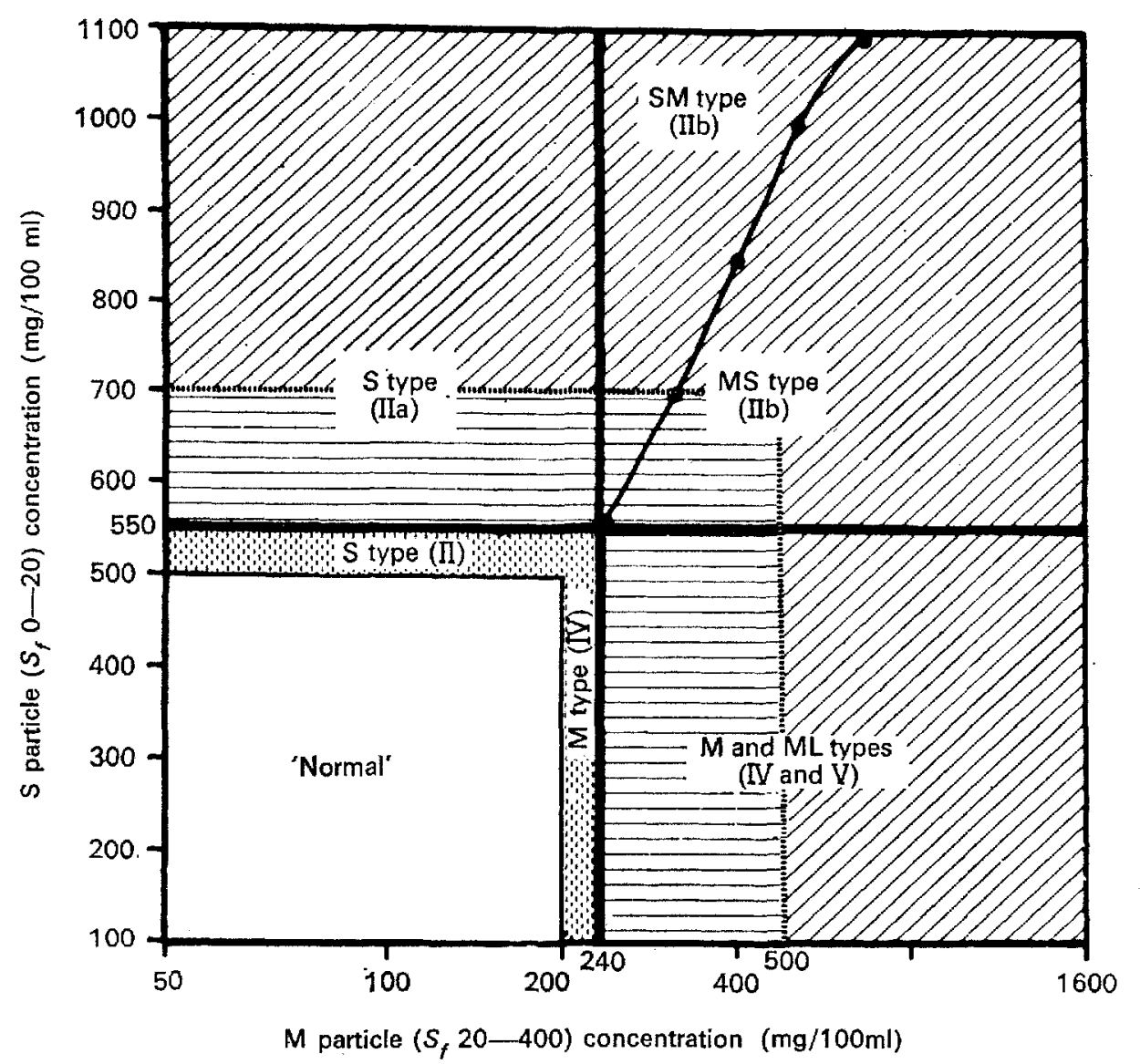

Fig. 1. Terminology and concentration limits for the various types of abnormal lipoprotein pattern. The vertical and horizontal lines separating the mild from the moderate area show the 99th percentile concentrations for $\mathrm{S}$ and $\mathrm{M}$ particles in young healthy subjects. (Stone et al. $197 \mathrm{x}$ ).

1, mild; $\boxminus$, moderate; $\square$, severe. 
abnormal SML type patterns are shown in Fig. I together with the appropriate terminology in the classification recommended by the WHO.

The diagnosis and treatment of hyperlipoproteinaemias can be based on the subject's lipoprotein pattern provided that sufficient information is available to assess its significance. To make an appropriate evaluation, the blood sample must be taken after a I 4 -h overnight fast and the subject must have been on his accustomed diet for at least 2 weeks. He must not be losing or gaining weight at the time of the examination and must not be taking any medication which might alter the lipoprotein concentrations. He should be examined for skin and tendon xanthomata, and a family history should be taken to assess the possibility of a familial hyperlipoproteinaemia. Some abnormal lipoprotein patterns occur as secondary manifestations of diseases such as untreated diabetes mellitus, obesity, myxoedema, nephrotic syndrome, and biliary cirrhosis; and these diseases should be excluded before a diagnosis of primary hyperlipoproteinaemia is made. Finally, because of the variability of the lipoprotein pattern in some subjects, at least two, and preferably three, analyses should be made at intervals of a few days.

The decision to treat a subject with an abnormal lipoprotein pattern will depend not only on the type of lipoprotein pattern, but on the circumstances under which it was found. If the abnormal pattern is secondary, then treatment of the primary disorder may reduce the lipoprotein concentrations to normal and no further treatment directed specifically at the hyperlipoproteinaemia is then required. If, however, the secondary hyperlipoproteinaemia does not completely resolve during treatment of the primary disease, or if the diagnosis of primary hyperlipoproteinaemia has been made, then the treatment chosen will depend on the variety of abnormal pattern. Three main groups of hyperlipoproteinaemias have been described using the SML classification and are termed S predominant, $M$ predominant and $\mathrm{L}$ predominant hyperlipoproteinaemias. Only the $\mathrm{S}$ and $\mathrm{M}$ predominant groups will be considered in this communication.

\section{$S$ predominant hyperlipoproteinaemias ( $S$ and SM types)}

The concentration limits for the $\mathrm{S}$ and SM types of hyperlipoproteinaemia are shown in Fig. 1. The presence of these patterns is associated with clear or faintly turbid serum with a raised concentration of serum cholesterol. Severely abnormal patterns are found in familial type II hyperlipoproteinaemias, and they are also found secondary to diseases such as nephrotic syndrome and myxoedema.

This is an important type of abnormality and is significantly more common in subjects with ischaemic heart disease than in apparently healthy subjects of the same age. In a random sample from a UK general practice, Stone et al. (I97I) found moderate and severe $\mathrm{S}$ predominant patterns in $5 \%$ of men and $1 \mathrm{I} \%$ of women aged $15-80$ years.

The concentration of $\mathbf{S}$ particles can be reduced to some extent by prescribing a diet in which the total fat is reduced and the percentage of saturated fat is only about one-third of the total fat ingested. The amount of cholesterol in the diet (normally about $700 \mathrm{mg} / \mathrm{d}$ ) should be reduced to below $300 \mathrm{mg} / \mathrm{d}$. We (Stone \& 
Dick, 1972) have found that the prevalence of $\mathrm{S}$ predominant patterns is significantly greater in obese males aged $3^{0-69}$ years than in their slim counterparts. It is often necessary therefore to use weight-reducing diets in the early stages of the treatment of type II hyperlipoproteinaemias. Three such diets are shown in Table 2. However, when a stable weight is reached the lipoprotein concentrations may rise once more to abnormal levels. The use of this type of diet will often result in a reduction of about $15 \%$ in the serum cholesterol, but in some subjects with a comparatively mild $\mathrm{S}$ type abnormality, a more marked reduction (up to $50 \%$ ) may occur.

Table 2. Composition of diets used in the treatment of $S$ predominant hyperlipoproteinaemia

$\begin{array}{cc}\text { (kcal) } & (\mathrm{MJ}) \\ 1200 & 5 \cdot 0 \\ 1500 & 6 \cdot 3 \\ 2000 & 8 \cdot 4\end{array}$

(Figures in parentheses show percentage of total energy)

$\begin{array}{cccc}\begin{array}{c}\text { Fat } \\ (\mathrm{g})\end{array} & \text { Carbohydrate } & \begin{array}{c}\text { Protein } \\ (\mathrm{g})\end{array} & \begin{array}{c}\text { Cholesterol } \\ (\mathrm{mg})\end{array} \\ 43(36) & (\mathrm{g}) & 66(24) & 200 \\ 57(36) & \mathrm{I} 2 \mathrm{I}(40) & 71(20) & 260 \\ 69(32) & \mathrm{I} 75(44) & 90(18) & 290\end{array}$

The majority of subjects with moderate or severe S predominant hyperlipoproteinaemias will not respond sufficiently well to diet alone, and drug therapy is usually required in addition. Among the drugs which have been used successfully in some subjects are cholestyramine - an anion exchange resin, thyroid hormones, neomycin, and clofibrate. Strisower, Adamson \& Strisower (I970), using clofibrate, have reported significant reductions in lipoprotein concentrations and we (M. C. Stone and T. B. S. Dick, unpublished) have seen one patient with severe heterozygous type II disease in whom a single xanthelasma completely disappeared, and there was a marked reduction in tendon xanthomas of the hands after treatment for 2 years with clofibrate and methyl clofenapate (a derivative of clofibrate (Thorp, I970)).

\section{$M$ predominant hyperlipoproteinaemias (MS, $M, M L$, and MIII types)}

The $M$ predominant hyperlipoproteinaemias are those in which the dominant feature in the lipoprotein pattern is the high concentration of M particles. It can be seen in Fig. I that this group includes MS, M and ML type patterns which correspond to types $I I_{b}, I V$ and $V$ in the WHO recommended classification. The $M$ predominant group also includes the MIII pattern which corresponds to Fredrickson type III hyperlipoproteinaemia. In a general practice random sample $12 \%$ of men and $4.5 \%$ of women had M predominant patterns (Stone et al. I97 I), the M type pattern being the most common of the four patterns in this group and the MIII type pattern being the least common.

The serum is turbid or milky in the postabsorptive phase due to light scattered by an increased concentration of medium-sized lipoproteins. If $\mathrm{L}$ particles are also present in significantly increased concentration, a layer of chylomicrons may be seen on the surface of the sample which has been left standing upright in a refrigerator 
at $4^{\circ}$ for $24-48 \mathrm{~h}$. The plasma triglyceride concentration is always raised (above $200 \mathrm{mg} / 100 \mathrm{ml}$ ) but cholesterol concentration may be low, normal or high depending on the type of pattern. The presence of an M predominant hyperlipoproteinaemia implies a defect in endogenous triglyceride transport which is carbohydrate-inducible, and the M type pattern is commonly found in association with abnormal glucose tolerance and also with hyperuricaemia.

There is a significant correlation between obesity and the prevalence of $\mathrm{M}$ predominant hyperlipoproteinaemias, especially $M$ type patterns (Stone \& Dick, 1972 ). For example, in men aged 30-69 years the prevalence of $M$ predominant hyperlipoproteinaemia was $5.6 \%$ in the slim tertile (based on subscapular skinfold thicknesses) and $24.8 \%$ in the obese tertile $(P<0.005)$. The first step in treating $M$ predominant hyperlipoproteinaemias is therefore to reduce the subject's weight to near the so-called ideal weight. A low-energy diet is used with about $35 \%$ of the energy derived from carbohydrate, and excluding alcohol and sugar. This will usually produce a significant weight reduction and a marked fall in M particle concentrations. However, once stable weight has been reached the lipoproteins may not remain at a normal level but may rise substantially as shown in Fig. 2. In these circumstances it is necessary to continue restriction of carbohydrate and alcohol, and also to use a drug such as clofibrate which will often maintain the concentrations at a satisfactory level.

Lipoprotein profiles obtained by quantitative analysis can be used for the planning of appropriate dietary therapy and to monitor the changes produced by treatment.

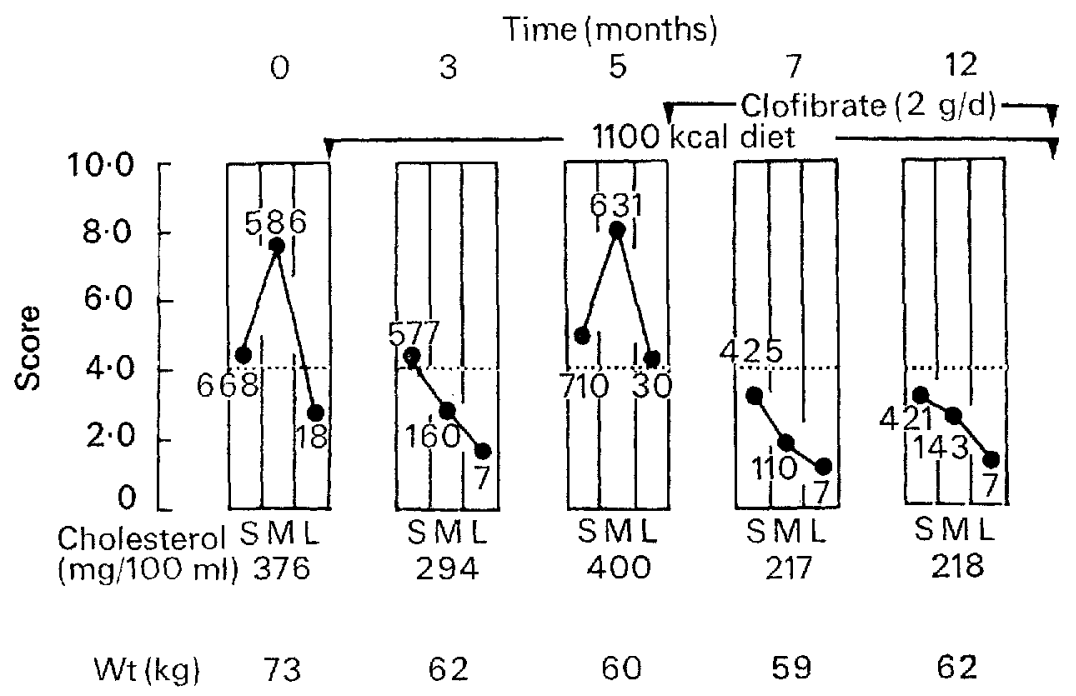

Fig. 2. Response of an MS type pattern to treatment with diet and clofibrate. The concentrations of the $\mathrm{S}, \mathrm{M}$ and $\mathrm{L}$ particle fractions are shown in $\mathrm{mg} / \mathrm{r} 00 \mathrm{ml}$. The dotted horizontal line is at the 'lower limit of significant abnormality' (i.e. the g9th percentile level in young healthy subjects). The scales of $S, M$ and $L$ particle concentrations have been transformed to 'scores' to eliminate the need for three separate scales. 


\section{REFERENCES}

Chin, H. P. \& Blankenhorn, D. H. (1968). Clinica chim. Acta 20, 305.

Fredrickson, D. S., Levy, R. I., Kwiterovich, P. O. Jr \& Jover, A. (I969). In Advances in Experimental Medicine and Biology Vol. 4. p. 307 [W. L. Holmes, L. A. Carlson and R. Paoletti, editors]. New York: Plenum Press.

Fredrickson, D. S., Levy, R. I. \& Lees, R. S. (1967). Nezw Engl. F. Med. 276, 34.

Hatch, F. T. \& Lees, R. S. (1968). In Advances in Lipid Research Vol. 6, p. 4 [R. Paoletti and D. Kritchevsky, editors]. New York: Academic Press.

Lees, R. S. \& Hatch, F. T. (1963). F. Lab. clin, Med. 6r, $5 \mathrm{I} 8$.

Masoro, E. J. (r 968$)$. Physiological Chemistry of Lipids in Mammals. Philadelphia: Saunders.

Noble, R. P., Hatch, F. T., Mazrimas, J. A., Lindgren, F. T., Jensen, L. C. \& Adamson, G. L. (1969). Lipids 4, 55 .

Stone, M. C. \& Dick, T. B. S. (1972). Eur. F. clin. Invest. (In the Press.)

Stone, M. C. \& Thorp, J. M. (1966). Clinica chim. Acta 14, 812.

Stone, M. C., Thorp, J. M., Mills, G. L. \& Dick, T. B. S. (1970). Clinica chim. Acta 30, 809 .

Stone, M. C., Thorp, J. M., Mills, G. L. \& Dick, T. B. S. (I97I). Clinica chim. Acta 3r, 333.

Strisower, E. H., Adamson, G. \& Strisower, B. (1970). Med. Clins N. America 54, I 599.

Thorp, J. M. (r970). In Atherosclerosis p. 54I [R. J. Jones, editor]. New York: Springer-Verlag.

Thorp, J. M., Horsfall, G. B. \& Stone, M. C. (1967). Med. biol. Engng 5, 5 I.

WHO (1970). Bull. Wld Hlth Org. 43, 8gr. 